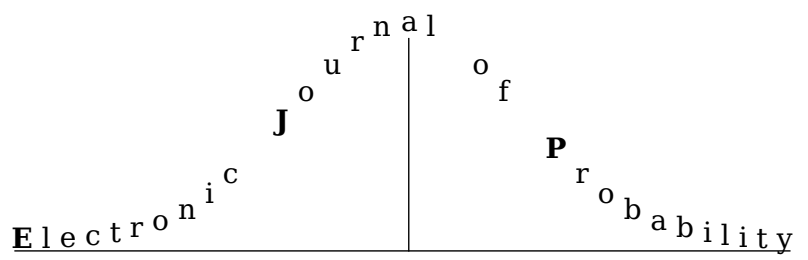

Electron. J. Probab. 27 (2022), article no. 35, 1-21.

ISSN: 1083-6489 https://doi.org/10.1214/22-EJP761

\title{
Spins, percolation and height functions
}

\author{
Marcin Lis*
}

\begin{abstract}
To highlight certain similarities in graphical representations of several well known two-dimensional models of statistical mechanics, we introduce and study a new family of models which specializes to these cases after a proper tuning of the parameters.

To be precise, our model consists of two independent standard Potts models, with possibly different numbers of spins and different coupling constants (the four parameters of the model), defined jointly on a graph embedded in a surface and its dual graph, and conditioned on the event that the primal and dual interfaces between spins of different value do not intersect. We also introduce naturally coupled height function and bond percolation models, and we discuss fundamental properties of the resulting joint coupling.

As special cases we recover the standard Potts and random cluster model, the six-vertex model and loop $O(n)$ model, the random current, double random current and XOR-Ising model.
\end{abstract}

Keywords: spin models; percolation; height functions.

MSC2020 subject classifications: 82B20.

Submitted to EJP on May 4, 2020, final version accepted on February 19, 2022.

\section{Introduction}

Graphical representations are ubiquitous in statistical mechanics and a thorough understanding of their interplay often leads to a transfer of information between different models. To capture some of their common features, we introduce a new family of twodimensional models with four parameters $\left(q, q^{\prime}, a, b\right) \in\{1,2, \ldots\}^{2} \times(0,1]^{2}$. To be precise, for a graph embedded in a surface, we consider three jointly coupled models (each of which comes in a primal and a dual version) in the form of

- spin models $\left(\sigma, \sigma^{\prime}\right)$ defined on the vertices and faces,

- bond percolation models $\left(\omega, \omega^{\prime}\right)$ on the primal and dual edges,

- height functions $\left(h, h^{\prime}\right)$ defined on the vertices and faces whose gradient is a deterministic and local function of $\left(\sigma, \sigma^{\prime}\right)$.

${ }^{*}$ University of Vienna E-mail: marcin. lis@univie.ac . at 
The starting point is the spin model which is given by a pair of independent primal and dual Potts models with $q$ and $q^{\prime}$ spins, and coupling constants satisfying $a=e^{-J}$ and $b=e^{-J^{\prime}}$ respectively, and conditioned on the event that their interfaces do not intersect (as we will see, this seemingly strange conditioning is the main reason why the model acquires some very natural properties). The percolation model is then built on top of the spin model using additional and independent randomness, and the height function is a deterministic function of the spin configuration. The relationship between the spin $\sigma$ and percolation model $\omega$ (and likewise for $\sigma^{\prime}$ and $\omega^{\prime}$ ) is a generalization of the EdwardsSokal coupling between Potts and Fortuin-Kasteleyn random cluster models $[16,17,36]$, meaning that $\sigma$ is obtained by independent assignments of spins to every cluster of $\omega$.

Our model includes as special cases the

- $\mathrm{FK}\left(q q^{\prime}\right)$ random cluster model for $a+b=1$,

- (staggered) six-vertex model for $q=q^{\prime}=2$,

- loop $O(n)$ model for $q=n, q^{\prime}=2$ and $b=1$,

- random current model for $q=1, q^{\prime}=2$ and $a^{2}+b^{2}=1$,

- double random current and XOR-Ising model for $q=q^{\prime}=2$ and $a^{2}+b^{2}=1$.

As a result, we give a unified framework for the known relations between these models [1, $6,9,16,19,22,30,31,35,37-39]$.

Moreover the coupling $\left(\omega, \omega^{\prime}, \sigma, \sigma^{\prime}, h, h^{\prime}\right)$ uncovers a new underlying structure. Indeed, another contribution of this article is the study of the stochastic interplay between the three different types of random configurations. For example, a crucial feature of this coupling will be that for $a+b \geq 1$, the two percolation configurations $\left(\omega, \omega^{\prime}\right)$ are such that an edge and its dual edge are never simultaneously open in $\omega$ and $\omega^{\prime}$ respectively (but they can be simultaneously closed). We call this the exlusion property. One should not confuse this with the exact exclusion property which says that an edge is present in $\omega$ if and only if its dual edge is absent in $\omega^{\prime}$. This stronger property holds true only on the line $a+b=1$ which corresponds to the classical random cluster model. On the other hand, the exclusion property does not hold for $a+b<1$. In this regime, an edge and its dual edge are never simultaneously closed in $\omega$ and $\omega^{\prime}$.

In the present work we use the exclusion property together with the rich structure of the law of $\left(\omega, \omega^{\prime}, \sigma, \sigma^{\prime}, h, h^{\prime}\right)$ that includes an Edwards-Sokal-type coupling between $\omega$ and $\sigma$, and the fact that the interfaces separating vertices with different values of $\sigma$ are contained in $\omega^{\prime}$ (and likewise for $\sigma^{\prime}$ and $\omega$ ). As a result we prove that the variance of the height function evaluated at a face is, up to constants, equal to the expected number of clusters in the percolation model $\omega$ that surround that face. This implies that in the self-dual model $a=b \geq 1 / 2$ on the square lattice (when $\omega$ and $\omega^{\prime}$ have the same distribution) the height function delocalizes (has unbounded variance) whenever $\omega$ does not percolate. Note that this is not a direct consequence since the exclusion property is not exact as in more classical percolation models. However, the structure of the coupling is rich enough to obtain this implication. By the Edwards-Sokal property, the fact that $\omega$ does not percolate, is up to technical details (that are for example taken care of in [29]), implied by the fact that $\sigma$ decorrelates at large distances. This is used in [29] to provide a proof of delocalization of the height function of the six-vertex model (that corresponds to $q=q^{\prime}=2$ ) for the range of parameters $a \in[1 / 2,1 / \sqrt{2+\sqrt{2}}]$. Before, the only rigorously known cases in this regime were $a=1 / 2$ [19], $a=\sqrt{2} / 2$ [9, 23], and its small neighbourhood [18], and $a=1$ [7]. Independently of [29] and using more technically involved arguments, the result was recently also extended in [15] to all $a \in[1 / 2,1]$. 
The coupling introduced in this paper is also crucially used in the recent proof of conformal invariance of critical double random currents $[13,14]$, and together with the construction of [28] should be relevant in the future study of the critical Ashkin-Teller model.

We note that in the special case $q=q^{\prime}=2$ and $a=b \leq 1 / 2$ the coupled percolation models $\left(\omega, \omega^{\prime}\right)$ studied here were independently introduced by Ray and Spinka in [37]. For $q=q^{\prime}=2$, the laws of the marginals on $\omega$ and $\omega^{\prime}$ (but not the full coupling) are also present in the work of Glazman and Peled [19] on the six-vertex model, and are closely related to the percolation models introduced by Pfister and Velenik [35]. We also note that the spin models $\left(\sigma, \sigma^{\prime}\right)$ are a variant of the interaction-round-a-face model studied in $[4,32]$.

This article is organized as follows:

- In Sect. 1 we define the model and describe the basic relationship between the spin, height function and percolation models.

- In Sect. 2 we study the interplay between the spin, percolation and height function model in more detail. We show an Edwards-Sokal-type coupling between the spins and the percolation clusters. Moreover we compare the variance of the difference of the height function between two points with the expected number of clusters of the percolation model that disconnect these points. Based on this property we establish in Theorem 2.8 that for the self-dual model on the square lattice $\left(q=q^{\prime}\right.$ and $\left.a=b \geq 1 / 2\right)$, the lack of percolation implies delocalization of the height function. In [29] we apply this the six-vertex model.

- In Sec. 3 we show how in special cases we recover other known models of statistical mechanics mentioned above. Moreover, using duality arguments, we provide an alternative representation of the planar spin model as a classical unconditional spin model which turns out to be a special case of the $\left(N_{\alpha}, N_{\beta}\right)$ model of Domany and Riedel [8]. This relation is a generalization of the Ashkin-Teller model representation of the six-vertex model $[31,39]$.

\section{The model}

The basis for our construction will be the Potts model. Let $Q$ be a finite set with $q$ elements. Recall that for a finite graph $G=(V, E)$ and a coupling constant $J$, the $q$-state Potts model [36] is a probability measure on $Q^{V}$ given by

$$
\mu(\mathbf{s})=\frac{1}{Z_{G, q}} \exp \left(-J \sum_{\left\{v_{1}, v_{2}\right\} \in E} \mathbf{1}\left\{\mathbf{s}\left(v_{1}\right) \neq \mathbf{s}\left(v_{2}\right)\right\}\right), \quad \mathbf{s} \in Q^{V},
$$

where $Z_{G, q}$ is the partition function. To denote the dependence on the parameter, we will write $Z_{G, q}=Z_{G, q}(x)$, where $x=e^{J}-1$. We say that the model is ferromagnetic if $J \geq 0$ (or equivalently $x \geq 0$ ) and antiferromagnetic if $J \leq 0(-1 \leq x \leq 0)$. We note that our definition is the standard one (see e.g. [21]) up to a rescaling of the weight in (1.1) by $e^{-J|E|}$.

The $q$-state Potts model is directly related to the $\mathrm{FK}(q)$ random cluster model [17] by the classical Edwards-Sokal coupling [16], where for each edge $\left\{v_{1}, v_{2}\right\}$ satisfying $\mathbf{s}\left(v_{1}\right)=\mathbf{s}\left(v_{2}\right)$, one declares it open with probability $1-e^{-J}$ and independently of other edges. The resulting configuration of open edges $\zeta$ gives rise to a bond percolation model which is the random cluster model. Moreover, in this coupling, conditioned on $\zeta$, the spins s can be recovered by choosing a uniform spin from $Q$ independently for each cluster of $\zeta$, i.e., a connected component of $(V, \zeta)$, including isolated vertices.

We are now ready to define our model. 


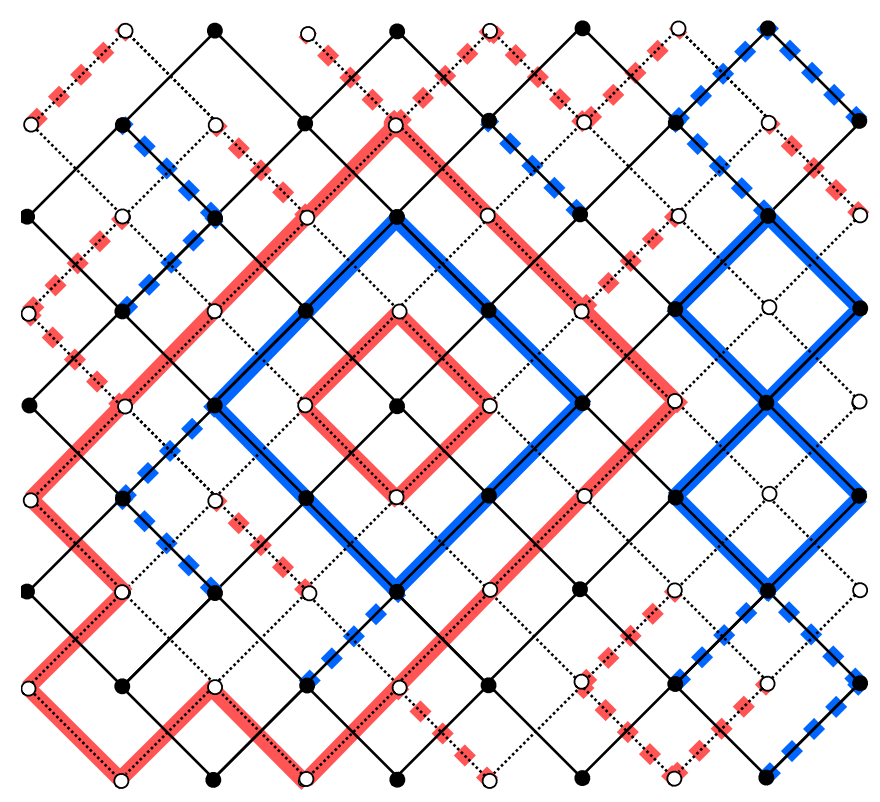

Figure 1: A piece of the square lattice (black vertices) and its dual (white vertices) together with a configuration satisfying the exclusion property $(a+b \geq 1)$. Note that the exclusion is not exact. The configurations $\omega$ and $\omega^{\prime}$ are drawn in blue and red respectively, and $\eta\left(\sigma^{\prime}\right) \subseteq \omega$ and $\eta(\sigma) \subseteq \omega^{\prime}$ are represented by solid lines. The spins $\sigma, \sigma^{\prime}$ and height functions $h, h^{\prime}$ are constant on the clusters of $\omega, \omega^{\prime}$ respectively. The solid lines are both domain walls (contours) for the spins and level lines for the height functions. In the coupling, the increments of the heigh function $h$ through different contours of $\eta\left(\sigma^{\prime}\right)$ are independent if the contours belong to different clusters of $\omega$, and otherwise they are deterministically related to each other

\subsection{Spin model}

Let $M$ be a compact, orientable surface with no boundary, or the plane. Let $\mathrm{G}=(\mathrm{V}, \mathrm{E})$ be a finite connected graph embedded in $M$ in such a way that each face is a topological disc, and let $\mathrm{G}^{*}=\left(\mathrm{U}, \mathrm{E}^{*}\right)$ be its dual also embedded in $M$, where $\mathrm{U}$ is identified with the set of faces of $\mathrm{G}$. For an edge $e \in \mathrm{E} \cup \mathrm{E}^{*}$, we write $e^{*} \in \mathrm{E} \cup \mathrm{E}^{*}$ for its dual edge. Similarly for $\omega \subseteq \mathrm{E} \cup \mathrm{E}^{*}$, we define $\omega^{*}=\left\{e^{*}: e \in \omega\right\}$.

Fix $q, q^{\prime} \in\{1,2, \ldots\}$ and choose two symmetric sets $Q, Q^{\prime} \subset \mathbb{C}$, i.e., satisfying $Q=-Q$ and $Q^{\prime}=-Q^{\prime}$, and such that $|Q|=q$ and $\left|Q^{\prime}\right|=q^{\prime}$. A spin configuration on $\mathrm{V}$ (resp. $\mathrm{U}$ ) is any function $\sigma: \mathrm{V} \rightarrow Q$ (resp. $\sigma^{\prime}: \mathrm{U} \rightarrow Q^{\prime}$ ). We define the contour configurations $\eta(\sigma) \subseteq$ $\mathrm{E}^{*}$ of $\sigma$ to be the set of all dual edges $e^{*}$ such that the endpoints of the corresponding primal edge $e$ are assigned different spin by $\sigma$. We also define $\eta\left(\sigma^{\prime}\right) \subseteq \mathrm{E}$ in a dual fashion. The configuration space of our (constrained) spin model is

$$
\Sigma=\left\{\left(\sigma, \sigma^{\prime}\right) \in Q^{\vee} \times{Q^{\prime}}^{\mathrm{U}}: \eta(\sigma)^{*} \cap \eta\left(\sigma^{\prime}\right)=\emptyset\right\} .
$$

In other words, this is the set of all pairs of primal and dual spin configurations $\left(\sigma, \sigma^{\prime}\right)$ whose interfaces, interpreted as subsets of $M$, do not intersect. Equivalently,

$$
\left(\sigma\left(v_{1}\right)-\sigma\left(v_{2}\right)\right)\left(\sigma^{\prime}\left(u_{1}\right)-\sigma^{\prime}\left(u_{2}\right)\right)=0
$$

for every pair of a primal edge $\left\{v_{1}, v_{2}\right\}$ and its dual $\left\{u_{1}, u_{2}\right\}$. 
We study a probability measure on $\Sigma$ given by

$$
\mathbf{P}\left(\sigma, \sigma^{\prime}\right)=\frac{1}{\mathcal{Z}} a^{\left|\eta\left(\sigma^{\prime}\right)\right|} b^{|\eta(\sigma)|},
$$

where $a, b \in(0,1]$ are parameters of the model, and $\mathcal{Z}=\mathcal{Z}\left(q, q^{\prime}, a, b\right)$ is the partition function. This measure is equivalent to a pair of independent primal and dual ferromagnetic Potts models with $q$ and $q^{\prime}$ spins, with coupling constants $J=-\log b$ and $J^{\prime}=-\log a$ respectively, and conditioned on $\Sigma$.

From this definition we immediately get the following description of mutual conditional laws for $\sigma$ and $\sigma^{\prime}$.

Corollary 1.1. Conditioned on the values of $\sigma^{\prime}$, the spins $\sigma$ are distributed like the $q$-state Potts model defined on the quotient graph $\mathrm{G} / \eta\left(\sigma^{\prime}\right)$ where each connected component of $\eta$ becomes a single vertex. By duality, the analogous statement holds true when the roles of $\sigma$ and $\sigma^{\prime}$ are exchanged.

Remark 1.2. We will only consider homogeneous weights $a$ and $b$, but most of our considerations generalize to non-homogeneous situations with $a_{e}=e^{-J_{e^{\prime}}^{\prime}}$ and $b_{e^{*}}=e^{-J_{e}}$, where $J$ and $J^{\prime}$ are arbitrary sets of positive coupling constants on the primal and dual edges respectively.

\subsection{The height function}

Assume that $M$ is of genus zero. We say that $\left\{v_{1}, u_{1}, v_{2}, u_{2}\right\}$ is a quad, if $\left\{v_{1}, v_{2}\right\} \in \mathrm{E}$ and $\left\{v_{1}, v_{2}\right\}^{*}=\left\{u_{1}, u_{2}\right\}$. For $\left(\sigma, \sigma^{\prime}\right) \in \Sigma$, we will consider a height function $H: \mathrm{V} \cup \mathrm{U} \rightarrow \mathbb{R}$ defined up to a constant by the rule: If $u \in \mathrm{U}$ and $v \in \mathrm{V}$ belong to the same quad, then

$$
H(u)-H(v)=\sigma(v) \sigma^{\prime}(u) .
$$

The constant can be chosen by fixing the value of the function at a particular vertex or face. That these relations are consistent follows from condition (1.3). Indeed, (1.3) is equivalent to the fact that the sum of the gradients (1.5) around each quad is zero. We will denote by $h$ and $h^{\prime}$ the restriction of $H$ to $\mathrm{V}$ and $\mathrm{U}$ respectively. Note that if $\left\{v_{1}, v_{2}\right\}$ and $\left\{u_{1}, u_{2}\right\}$ are mutually dual edges, then

$$
\begin{aligned}
h\left(v_{2}\right)-h\left(v_{1}\right) & =\sigma^{\prime}\left(u_{1}\right)\left(\sigma\left(v_{2}\right)-\sigma\left(v_{1}\right)\right)=\sigma^{\prime}\left(u_{2}\right)\left(\sigma\left(v_{2}\right)-\sigma\left(v_{1}\right)\right), \\
h^{\prime}\left(u_{2}\right)-h^{\prime}\left(u_{1}\right) & =\sigma\left(v_{1}\right)\left(\sigma^{\prime}\left(u_{2}\right)-\sigma^{\prime}\left(u_{1}\right)\right)=\sigma\left(v_{2}\right)\left(\sigma^{\prime}\left(u_{2}\right)-\sigma^{\prime}\left(u_{1}\right)\right) .
\end{aligned}
$$

It follows from the definition that $h$ is constant on the clusters of constant spin $\sigma$, and $h^{\prime}$ is constant on the clusters of constant spin $\sigma^{\prime}$.

Remark 1.3. For surfaces of higher genus one can define in the same way a height function on the universal cover of $M$. Equivalently, one can talk about the increment of the height function between two points taken along a curve, up to the homotopy of the curve. We will use the latter definition applied to the torus in Theorem 2.8.

\subsection{Bond percolation}

We also augment the model with a bond percolation configuration using the following procedure: Given $\left(\sigma, \sigma^{\prime}\right) \in \Sigma$ sampled according to $\mathbf{P}$,

1. Declare each primal edge in $\eta\left(\sigma^{\prime}\right)$ and each dual edge in $\eta(\sigma)$ open. This is to say that an edge is open if the corresponding dual edge carries two different spins in the dual spin configuration.

2. For each pair of a primal and its dual edge $e$ and $e^{*}$ such that neither $e \in \eta\left(\sigma^{\prime}\right)$ nor $e^{*} \in \eta(\sigma)$, and independently of other such pairs, declare the state of the edges with the following probabilities chosen depending on the value of $a+b$ : 
Spins, percolation and height functions

\begin{tabular}{|c|c|c|}
\cline { 2 - 3 } \multicolumn{1}{c|}{} & $a+b \leq 1$ & $a+b \geq 1$ \\
$e$ open, $e^{*}$ closed & $a$ & $1-b$ \\
blosed, $e^{*}$ open & $b$ & $1-a$ \\
both $e, e^{*}$ open & $1-a-b$ & 0 \\
\hline
\end{tabular}

Note that in both cases the probability of opening $e$ and $e^{*}$ is $1-b$ and $1-a$ respectively.

We call the resulting set of all open primal and dual edges $\omega$ and $\omega^{\prime}$ respectively. Note that $\omega \backslash \eta\left(\sigma^{\prime}\right)$ is exactly the set of open edges from the Edwards-Sokal coupling mentioned above applied to the Potts model $\sigma$ on the quotient graph $\mathrm{G} / \eta\left(\sigma^{\prime}\right)^{1}$. By duality, the same is true for $\omega^{\prime} \backslash \eta(\sigma)$ and $\sigma^{\prime}$.

A cluster of $\omega$, resp. $\omega^{\prime}$, is a connected component of the graph $(\mathrm{V}, \omega)$, resp. $\left(\mathrm{U}, \omega^{\prime}\right)$, including the isolated vertices. We define

$$
\begin{array}{r}
\Omega \Sigma=\left\{\left(\omega, \omega^{\prime}, \sigma, \sigma^{\prime}\right): \sigma \text { constant on clusters of } \omega \text { and } \eta(\sigma) \subseteq \omega^{\prime},\right. \\
\left.\sigma^{\prime} \text { constant on clusters of } \omega^{\prime} \text { and } \eta\left(\sigma^{\prime}\right) \subseteq \omega\right\},
\end{array}
$$

where $\left(\sigma, \sigma^{\prime}\right) \in \Sigma$, to be the space of consistent configurations for the spin model augmented with the sets of open edges, and we denote by $\mathbf{P}\left(\omega, \omega^{\prime}, \sigma, \sigma^{\prime}\right)$ the probability measure on $\Omega \Sigma$ given by the coupling above.

Remark 1.4. We note that the left-hand side of the table above for $q=q^{\prime}=2$ and $a=b$ describes the process studied by Ray and Spinka [37].

For $\xi \subseteq \mathrm{E}$, we write $\xi^{\dagger}=\mathrm{E}^{*} \backslash \xi^{*}$. It follows from the definition that

$$
\omega^{\dagger} \subseteq \omega^{\prime} \text { for } a+b \leq 1, \quad \text { and } \quad \omega^{\dagger} \supseteq \omega^{\prime} \text { for } a+b \geq 1
$$

almost surely. We define $\Omega \Sigma_{\leq 1}$ and $\Omega \Sigma_{\geq 1}$ respectively to be $\Omega \Sigma$ with these additional restrictions imposed on $\omega$ and $\omega^{\prime}$.

Note that if $\left(\omega, \omega^{\prime}, \sigma, \sigma^{\prime}\right) \in \Omega \Sigma_{\geq 1}$, then $\omega$ and $\omega^{\prime}$ satisfy the mentioned exclusion property. Moreover for the boundary case $a+b=1$, we have exact exclusion meaning that $\omega^{\dagger}=\omega^{\prime}$ almost surely.

Finally, by definition the weight of each configuration $\left(\omega, \omega^{\prime}, \sigma, \sigma^{\prime}\right) \in \Omega \Sigma_{\leq 1}$ is

$$
a^{\left|\omega^{*} \backslash \omega^{\prime}\right|} b^{\left|\omega^{\prime} \backslash \omega^{*}\right|}(1-a-b)^{\left|\omega^{*} \cap \omega^{\prime}\right|},
$$

and the weight of $\left(\omega, \omega^{\prime}, \sigma, \sigma^{\prime}\right) \in \Omega \Sigma_{\geq 1}$ is

$$
a^{\left|\eta\left(\sigma^{\prime}\right)\right|}(1-b)^{\left|\omega \backslash \eta\left(\sigma^{\prime}\right)\right|} b^{|\eta(\sigma)|}(1-a)^{\left|\omega^{\prime} \backslash \eta(\sigma)\right|}(a+b-1)^{\mathrm{E}^{*} \backslash\left(\omega^{*} \cup \omega^{\prime}\right)} .
$$

Note that (1.8) is independent of $\left(\sigma, \sigma^{\prime}\right)$.

\section{Properties of the coupling}

In this section we discuss fundamental properties of the coupling between the three types of random configurations.

\subsection{Edwards-Sokal property}

It turns out that the clusters of $\omega$ encode geometrically the stochastic dependencies of the spin model $\sigma$. This is manifested in the following Edwards-Sokal property of the coupling between $\sigma$ and $\omega$. Clearly, the same holds for $\omega^{\prime}$ and $\sigma^{\prime}$ by duality. In the following, for $\xi \subseteq \mathrm{E}$, we denote by $V(\xi)$ the set of vertices incident on at least one edge in $\xi$.

\footnotetext{
${ }^{1}$ We thank Ron Peled for this observation
} 
Proposition 2.1. Conditioned on the configuration $\omega$,

1. the spin $\sigma$ is distributed like an independent uniform assignment of a spin from $Q$ to each cluster of $\omega$.

2. the spin $\sigma^{\prime}$ is distributed like the $q^{\prime}$-state Potts model with coupling constant $J$ satisfying $e^{-J}=\frac{a}{1-b}$, and defined on the dual $(\mathrm{V}(\omega), \omega)^{*}$ of $(\mathrm{V}(\omega), \omega)$, i.e., the graph whose vertices are the faces of $\omega$ and where two faces are adjacent, if they share an edge in $\omega$. Note that multiple edges and loops are possible.

3. in particular, $\sigma$ and $\sigma^{\prime}$ are independent.

Proof. We claim that for fixed $\left(\omega, \sigma^{\prime}\right)$ with $\eta\left(\sigma^{\prime}\right) \subseteq \omega$, the weight of each consistent configuration $\left(\omega, \sigma, \sigma^{\prime}\right)$, i.e., such that $\sigma$ is constant on the clusters of $\omega$, is equal to

$$
a^{\left|\eta\left(\sigma^{\prime}\right)\right|}(1-b)^{\left|\omega \backslash \eta\left(\sigma^{\prime}\right)\right|} b^{\left|\omega^{\dagger}\right|},
$$

where $\omega^{\dagger}=\mathrm{E}^{*} \backslash \omega^{*}$, and in particular is independent of $\sigma$. Indeed this follows from the fact that each edge in

- $\eta(\sigma)$ contributes weight $b$ by the definition of the spin model,

- $\omega^{\dagger} \backslash \eta(\sigma)$ also contributes weight $b$ since this is the probability that a dual edge $\left\{u_{1}, u_{2}\right\}$ with $\sigma^{\prime}\left(u_{1}\right)=\sigma^{\prime}\left(u_{2}\right)$ ends up in $\omega^{\dagger}$ in step (2) of the definition of the edge percolation model.

This means that conditioned on $\left(\omega, \sigma^{\prime}\right)$, we have a uniform distribution on all spin configurations $\sigma$ such that $\eta(\sigma) \subseteq \omega^{\dagger}$. This is equivalent to choosing an independent uniform spin for each cluster of $\omega$ and we conclude property (1).

Property (2) also follows from (2.1), the definition of the Potts model, and the fact that the only constraint on $\sigma^{\prime}$ is that $\eta\left(\sigma^{\prime}\right) \subseteq \omega$.

Conditional independence of $\sigma$ and $\sigma^{\prime}$ follows from the fact that (2.1) does not depend on $\sigma$.

We note that property (1) for $q=q^{\prime}=2$ was first studied in [19, 37].

Remark 2.2. Since (1.8) is independent of both $\sigma$ and $\sigma^{\prime}$ for $a+b \leq 1$, property (1) from the proposition above holds in this case simultaneously for $\sigma$ and $\sigma^{\prime}$ when conditioned on $\left(\omega, \omega^{\prime}\right)$.

We start by showing how $\sigma$-spin correlations are described by $\omega$-connectivity probabilities in the same way the Potts model correlations are given by the random cluster connectivity probabilities (the Edwards-Sokal property). Let $\sigma_{0}$ be a random variable uniformly distributed on $Q$, and let $m(k)=\left\langle\sigma_{0}^{k}\right\rangle$ for $k \in\{0,1, \ldots\}$. Since we assume that $Q$ is a symmetric subset of $\mathbb{R}$, we have $m(2 k+1)=0$. For $\xi \subseteq \mathrm{E}$ and $v_{1}, \ldots, v_{k} \in \mathrm{V}$, we define $\pi(\xi)$ to be the partition of $\left\{v_{1}, \ldots, v_{k}\right\}$ induced by the connected components of $\xi$.

Proposition 2.3 ( $\sigma$-spin correlations via $\omega$-connectivities). Let $v_{1}, \ldots, v_{k} \in \mathrm{V}$ and $r_{1}, \ldots$, $r_{k} \in\{0,1, \ldots\}$. Then

$$
\mathbf{E}\left[\sigma\left(v_{1}\right)^{r_{1}} \cdots \sigma\left(v_{k}\right)^{r_{k}}\right]=\sum_{\mathcal{P}} \mathbf{P}(\pi(\omega)=\mathcal{P}) \prod_{A \in \mathcal{P}} m\left(\sum_{v_{j} \in A} r_{j}\right),
$$

where the sum is taken over all partitions $\mathcal{P}$ of $\left\{v_{1}, \ldots, v_{k}\right\}$ such that for all $A \in \mathcal{P}$, $\sum_{v_{j} \in A} r_{j}$ is even. In particular,

$$
\mathbf{E}\left[\sigma\left(v_{1}\right) \sigma\left(v_{2}\right)\right]=m(2) \mathbf{P}\left(v_{1} \stackrel{\omega}{\leftrightarrow} v_{2}\right),
$$

where $\left\{v_{1} \stackrel{\omega}{\leftrightarrow} v_{2}\right\}$ is the event that $v_{1}$ and $v_{2}$ are in the same cluster of $\omega$.

Proof. It is enough to condition on $\omega$ and use Proposition 2.1. 


\subsection{Height function variance via nesting of percolation clusters}

We will now compare the variance of the difference of the height function $h^{\prime}$ between two faces $u_{1}$ and $u_{2}$ to the expected number of clusters of the percolation models that one has to cross when going from $u_{1}$ to $u_{2}$. We assume here that $M$ is of genus zero but similar arguments taking into account nontrivial topology of some clusters can be applied to surfaces of higher genus.

Consider the percolation configuration $\omega$, and let $\sigma$ and $\sigma^{\prime}$ be consistent with $\omega$, i.e., $\sigma$ is constant on the clusters of $\omega$ and $\eta\left(\sigma^{\prime}\right) \subseteq \omega$. For a cluster $\mathcal{C}$ of $\omega$, define $\sigma_{\mathcal{C}}^{\prime}: \mathrm{U} \rightarrow Q^{\prime}$ to be the spin configuration which is equal to $\sigma^{\prime}$ on the faces incident on $\mathcal{C}$ and satisfies $\eta\left(\sigma_{\mathcal{C}}^{\prime}\right) \subseteq \mathcal{C}$. In other words, $\sigma_{\mathcal{C}}^{\prime}$ is the spin configuration obtained from $\sigma^{\prime}$ by erasing the contours in $\eta\left(\sigma^{\prime}\right)$ which are not contained in $\mathcal{C}$ and changing the spins in a consistent way. Also, denote by $\sigma(\mathcal{C})$ the value of $\sigma$ assigned to any vertex in $\mathcal{C}$. Fix $u_{1}, u_{2} \in \mathrm{U}$, and let

$$
d h^{\prime}=h\left(u_{2}\right)-h\left(u_{1}\right), \quad \text { and } \quad d \sigma_{\mathcal{C}}^{\prime}=\sigma_{\mathcal{C}}^{\prime}\left(u_{2}\right)-\sigma_{\mathcal{C}}^{\prime}\left(u_{1}\right)
$$

We claim that

$$
d h^{\prime}=\sum_{\mathcal{C}} \sigma(\mathcal{C}) d \sigma_{\mathcal{C}}^{\prime}
$$

To justify this, we will say that a non-trivial cluster $\mathcal{C}$ of $\omega$ disconnects $u_{1}$ from $u_{2}$, if the two faces belong to two different connected components of $M \backslash \mathcal{C}$, where we think of $\mathcal{C}$ as the closed subset of $M$ given by the union of its edges.

Remark 2.4. Note that if $\mathcal{C}$ does not disconnect $u_{1}$ from $u_{2}$, then $d \sigma_{\mathcal{C}}^{\prime}=0$. Actually if $d \sigma_{\mathcal{C}}^{\prime} \neq 0$, then $\mathcal{C}$ necessarily contains a connected component of $\eta\left(\sigma^{\prime}\right)$ that disconnects $u_{1}$ from $u_{2}$.

By the above remark, the sum in (2.2) can be restricted to only these clusters that disconnect $u_{1}$ from $u_{2}$. Let $\gamma=\left\{\tilde{u}_{1}, \ldots, \tilde{u}_{l}\right\}$ be a path of pairwise adjacent faces with $\tilde{u}_{1}=u_{1}$ and $\tilde{u}_{l}=u_{2}$, and such that the only non-trivial clusters of $\omega$ that it crosses are those that disconnect $u_{1}$ from $u_{2}$, and moreover $\gamma$ crosses each of these clusters only once. For $j \in\{1, \ldots, l-1\}$, let $v_{j}$ be one of the two vertices of the edge dual to $\left\{\tilde{u}_{j}, \tilde{u}_{j+1}\right\}$. By the definition of $h^{\prime}(1.7)$ we have

$$
d h^{\prime}=\sum_{j=1}^{l} \sigma\left(v_{j}\right)\left(\sigma^{\prime}\left(\tilde{u}_{j}\right)-\sigma^{\prime}\left(\tilde{u}_{j+1}\right)\right) .
$$

Note that as long as $\gamma$ stays in between two clusters of $\omega$, the increments in the sum above are zero since the value of $\sigma^{\prime}\left(\tilde{u}_{j}\right)$ is constant. On the other hand, when $\gamma$ crosses a cluster $\mathcal{C}$, then the value of $\sigma\left(v_{j}\right)$ is constant and equal to $\sigma(\mathcal{C})$, and hence, by a telescopic sum, the contribution corresponding to $\mathcal{C}$ is exactly $\sigma(\mathcal{C}) d \sigma_{\mathcal{C}}^{\prime}$. This justifies (2.2).

We will now use this formula to estimate the variance of $d h^{\prime}$. To this end, let $\sigma_{0}$ be a random variable uniformly distributed on $Q$. By property (1) and (2) from Proposition 2.1, conditioned on $\omega, \sigma(\mathcal{C})$ and $\sigma_{\mathcal{C}}^{\prime}$ are independent, and moreover $\sigma(\mathcal{C}) \sim \sigma_{0}$ and $\sigma\left(\mathcal{C}^{\prime}\right) \sim \sigma_{0}$ are independent for different clusters $\mathcal{C}$ and $\mathcal{C}^{\prime}$ of $\omega$. Hence, using (2.2) we can write

$$
\begin{aligned}
\operatorname{Var}\left[d h^{\prime}\right] & =\mathbf{E}\left[\left(\sum_{\mathcal{C}} \sigma(\mathcal{C}) d \sigma_{\mathcal{C}}^{\prime}\right)^{2}\right] \\
& =\sum_{\omega \subseteq \mathrm{E}} \sum_{\mathcal{C}_{1}, \mathcal{C}_{2} \subseteq \omega} \mathbf{E}\left[\sigma\left(\mathcal{C}_{1}\right) d \sigma_{\mathcal{C}_{1}}^{\prime} \sigma\left(\mathcal{C}_{2}\right) d \sigma_{\mathcal{C}_{2}}^{\prime} \mid \omega\right] \mathbf{P}(\omega) \\
& =\sum_{\omega \subseteq \mathrm{E}} \sum_{\mathcal{C}_{1}, \mathcal{C}_{2} \subseteq \omega} \mathbf{E}\left[\sigma\left(\mathcal{C}_{1}\right) \sigma\left(\mathcal{C}_{2}\right) \mid \omega\right] \mathbf{E}\left[d \sigma_{\mathcal{C}_{1}}^{\prime} d \sigma_{\mathcal{C}_{2}}^{\prime} \mid \omega\right] \mathbf{P}(\omega)
\end{aligned}
$$




$$
\begin{aligned}
& =\mathbf{E}\left[\sigma_{0}^{2}\right] \sum_{\omega \subseteq \mathbb{E}} \sum_{\mathcal{C} \subseteq \omega} \mathbf{E}\left[\left(d \sigma_{\mathcal{C}}^{\prime}\right)^{2} \mid \omega\right] \mathbf{P}(\omega) \\
& =\mathbf{E}\left[\sigma_{0}^{2}\right] \mathbf{E}\left[\sum_{\mathcal{C}}\left(d \sigma_{\mathcal{C}}^{\prime}\right)^{2}\right] \\
& =\mathbf{E}\left[\sigma_{0}^{2}\right] \sum_{d \neq 0} d^{2} \mathbf{E}\left[N_{d}\right]
\end{aligned}
$$

where $N_{d}=N_{d}\left(u_{1}, u_{2}\right)$ is the number of clusters $\mathcal{C}$ of $\omega$ such that $d \sigma_{\mathcal{C}}^{\prime}=d$. Define $N_{\neq 0}=\sum_{d \neq 0} N_{d}$, and let $C / 2=\max \left\{|i|: i \in Q^{\prime}\right\}$. Then $\left|d \sigma_{\mathcal{C}}^{\prime}\right| \leq C$, and from the above computation we immediately get the following equivalence up to constants.

Proposition 2.5. We have

$$
C^{2} \mathbf{E}\left[\sigma_{0}^{2}\right] \mathbf{E}\left[N_{\neq 0}\right] \geq \operatorname{Var}\left[d h^{\prime}\right] \geq \mathbf{E}\left[\sigma_{0}^{2}\right] \mathbf{E}\left[N_{\neq 0}\right] .
$$

Note that in the special case when $q^{\prime}=2$ and $Q^{\prime}=\{-i, i\}$, we actually get the equality

$$
\operatorname{Var}\left[d h^{\prime}\right]=4 i^{2} \mathbf{E}\left[\sigma_{0}^{2}\right] \mathbf{E}\left[N_{\neq 0}\right] .
$$

We note that this identity in the setting of the double random current model and the related height function was first obtained in [12], where it was used to establish continuity of the phase transition of the Ising model on any bi-periodic planar graph.

To prove the final result of this section we will need the following description of the mutual conditional laws for the two bond percolation processes.

Lemma 2.6. Assume that $a+b \geq 1$. Then conditioned on $\left(\omega^{\prime}, \sigma^{\prime}\right)$, the percolation configuration $\omega$ is distributed like $\eta\left(\sigma^{\prime}\right) \cup \zeta$, where $\zeta$ is an independent bond percolation process on $\left(\omega^{\prime}\right)^{\dagger}$ with success probability $(1-b) / a$.

Proof. This follows from the fact that, tossing the four-sided die from the right-hand side of the table in (1), and conditioning on $e^{*}$ being closed, we open $e$ with probability $(1-b) / a$.

We say that a cluster $\mathcal{C}^{\prime}$ of $\omega^{\prime}$ disconnects face $u_{1}$ from $u_{2}$, if either one of the two faces is in $\mathcal{C}^{\prime}$, or they belong to two different connected components of $M \backslash \mathcal{C}^{\prime}$, where we think of $\mathcal{C}^{\prime}$ as the closed subset of $M$ given by the union of its edges. Note that the trivial clusters containing $u_{1}$ and $u_{2}$ satisfy this definition.

The next result compares the number of clusters of $\omega^{\prime}$ disconnecting $u_{1}$ from $u_{2}$ with the number of clusters $\mathcal{C}$ of $\omega$ which satisfy $d \sigma_{\mathcal{C}}^{\prime} \neq 0$ in the case $a+b \geq 1$.

Proposition 2.7. Let $N^{\prime}=N^{\prime}\left(u_{1}, u_{2}\right)$ be the number of clusters of $\omega^{\prime}$, that disconnect $u_{1}$ from $u_{2}$. Assume that $a+b \geq 1$. Then

$$
\mathbf{E}\left[N_{\neq 0}\right] \geq\left(1-\frac{1}{q^{\prime}}\right)\left(\mathbf{E}\left[N^{\prime}\right]-1\right) .
$$

Proof. Recall that by Proposition 2.1, when conditioned on $\omega^{\prime}$, the spins $\sigma^{\prime}$ are assigned to each cluster of $\omega^{\prime}$ independently and uniformly in $Q^{\prime}$. Let $\mathcal{C}_{1}^{\prime}, \ldots, \mathcal{C}_{N^{\prime}}^{\prime}$ be the clusters of $\omega^{\prime}$ that disconnect $u_{1}$ from $u_{2}$ ordered according to the first intersection points with a chosen path from $u_{1}$ to $u_{2}$. Note that if two consecutive clusters $\mathcal{C}_{l}^{\prime}, \mathcal{C}_{l+1}^{\prime}$ are assigned different spins, then for topological reasons, there must exist a circuit in $\eta\left(\sigma^{\prime}\right)$ disconnecting $\mathcal{C}_{i}^{\prime}$ from $\mathcal{C}_{l+1}^{\prime}$, and hence also disconnecting $u_{1}$ from $u_{2}$.

By Lemma 2.6, conditioned on $\sigma^{\prime}$ and $\omega^{\prime}$, we recover $\omega$ by choosing randomly edges from $\left(\omega^{\prime}\right)^{\dagger}$ and adding them to $\eta\left(\sigma^{\prime}\right)$. This means that for every pair $\mathcal{C}_{l}^{\prime}, \mathcal{C}_{l+1}^{\prime}$ with different spin $\sigma^{\prime}$, there exists at least one cluster $\mathcal{C}$ of $\omega$, disconnecting $u_{1}$ from $u_{2}$. Moreover, at least one of these clusters must satisfy $d \sigma_{\mathcal{C}}^{\prime}\left(u_{1}, u_{2}\right) \neq 0$ (since the sum of $d \sigma_{\mathcal{C}}^{\prime}$ over all such clusters is nonzero). Since the clusters of $\omega^{\prime}$ are disjoint, the clusters of $\omega$ corresponding 
to different pairs $\mathcal{C}_{l}^{\prime}, \mathcal{C}_{l+1}^{\prime}$ are also disjoint. This means that $N_{\neq 0}$ is at least equal to the number of pairs $\mathcal{C}_{l}^{\prime}, \mathcal{C}_{l+1}^{\prime}$ with different spin $\sigma^{\prime}$. The latter is equal in distribution to the number of nearest neighbour disagreements in an i.i.d. sequence of length $N^{\prime}$ and distribution $\sigma_{0}$. Hence, we get the desired inequality by an elementary computation of the expectation.

\section{Application to the self-dual model with $q=q^{\prime}$ and $a=b$}

For $q=q^{\prime}=2$, the question of delocalization at $a=b>1 / 2$ is still open for a large range of $a$. One of the difficulties in studying this regime is that, e.g. the classical Baxter-Kelland-Wu coupling [3] between the six-vertex model and the random cluster model is no longer a probabilistic construction but involves complex-valued measures. In our case this is the regime where the marginal of $\mathbf{P}$ on $\omega$ is not positively associated which is a major technical obstacle. We say that a bond percolation process on an infinite graph percolates if it contains an infinite cluster. The main contribution of this section is a partial result saying that no percolation of $\omega$ is a sufficient condition for delocalization in the self-dual model.

To make full use of translation invariance, we will consider the model $\mathbf{P}_{\mathbb{T}_{n}}$ defined on the square lattice torus $\mathbb{T}_{n}$ of size $n \times n$. Self-duality implies that $\omega$ shifted by $\left(\frac{1}{2}, \frac{1}{2}\right)$ (so that $\mathbb{T}_{n}$ becomes $\mathbb{T}_{n}^{*} \simeq \mathbb{T}_{n}$ ) has the same distribution under $\mathbf{P}_{\mathbb{T}_{n}}$ as $\omega^{\prime}$. This property clearly carries over to any subsequential limit $\mathbf{P}_{\mathbb{Z}^{2}}=\lim _{k \rightarrow \infty} \mathbf{P}_{\mathbb{T}_{n_{k}}}$. We talk about subsequential limits here since there is no evident stochastic monotonicity of the model that would guarantee the uniqueness of the limit. In our last theorem we show that if $\omega$ does not percolate $\mathbf{P}_{\mathbb{Z}^{2}}$-almost surely, then $\omega$ necessarily contains infinitely many clusters surrounding the origin and hence the associated height function delocalizes. The first implication is immediate by self-duality whenever $\omega^{\dagger} \subseteq \omega^{\prime}$, but this property only holds for $a=b \leq 1 / 2$. To get the result in full generality we use the essential fact that $\eta(\sigma) \subseteq \omega^{\prime}$, which in particular implies that if $\eta(\sigma)$ percolates, then so does $\omega^{\prime}$.

Theorem 2.8. Consider a subsequential limit $\mathbf{P}_{\mathbb{Z}^{2}}=\lim _{k \rightarrow \infty} \mathbf{P}_{\mathbb{T}_{n_{k}}}$ of the self-dual model with $q=q^{\prime}$ and $a=b \geq 1 / 2$, and assume that

$$
\mathbf{P}_{\mathbb{Z}^{2}}(\omega \text { percolates })=0 .
$$

Then

$$
\left.\mathbf{P}_{\mathbb{Z}^{2}} \text { (infinitely many clusters of } \omega \text { surround the origin }\right)=1 \text {. }
$$

and

$$
\lim _{\left|u_{1}-u_{2}\right| \rightarrow \infty} \operatorname{Var}_{\mathbf{P}_{\mathbb{Z}^{2}}}\left[h^{\prime}\left(u_{1}\right)-h^{\prime}\left(u_{2}\right)\right]=\infty .
$$

We note that the particular choice of the path in the statement above is not essential to the argument.

Remark 2.9. Since $a=b>\frac{1}{2}$, we have the exclusion property $\omega^{*} \cap \omega^{\prime}=\emptyset$. One therefore expects (2.3) to hold true if e.g. one can establish ergodicity of the marginal of $\mathbf{P}_{\mathbb{Z}^{2}}$ onto $\omega$. Indeed, if this were true, then by duality, positive probability of percolation would imply coexistence of disjoint infinite clusters of $\omega$ and $\omega^{\prime}$, which one does not expect to happen. However, it is not clear why the infinite volume limit should be ergodic, and indeed it follows from the results of [11] and Proposition 3.6 that this is not true in the boundary case $a=1 / 2$ and $q>2$.

To prove the theorem, we will need the following lemma. It is highly likely that this result exists in the literature but we could not find a proper reference, and thus we give a proof for completeness. 
Lemma 2.10. Condition (2.3) guarantees that property (1) from Proposition 2.1, which says that under $\mathbf{P}_{\mathbb{T}_{n_{k}}}$ the spins $\sigma$ are sampled by independently chosing a spin for each cluster of $\omega$, carries over into the infinite volume limit $\mathbf{P}_{\mathbb{Z}^{2}}$.

Proof. Let $\mathbf{S}$ be the law on spin configurations on the vertices of $\mathbb{Z}^{2}$ coupled with $\mathbf{P}_{\mathbb{Z}^{2}}$ by independently assigning a spin from $Q$ to each cluster of $\omega$. We need to prove that $\mathbf{S}$ is equal in distribution to the marginal of $\mathbf{P}_{\mathbb{Z}^{2}}$ on $\sigma$. To this end, consider a fixed box $\Lambda_{l}$ and $\varepsilon>0$. For $L>l$, let $E_{l, L}$ be the event that no cluster of $\omega$ connects $\Lambda_{l}$ to the boundary of $\Lambda_{L}$, and take $L$ so large that $\mathbf{P}_{\mathbb{Z}^{2}}\left(E_{l, L}\right) \geq 1-\varepsilon$. Such $L$ exists since by (2.3) there are only finite clusters almost surely. Next, take $k$ so large that $n_{k}>L$ and the total variation distance between the law of $\mathbf{P}_{\mathbb{T}_{n_{k}}}$ and $\mathbf{P}_{\mathbb{Z}^{2}}$ restricted to $\Lambda_{L}$ is smaller than $\varepsilon$. This is possible by weak convergence and since $\Lambda_{L}$ is fixed. Let $\mathbf{P}_{1}$ be the former and $\mathbf{P}_{2}$ the latter law, and denote by $\left(\omega_{1}, \sigma_{1}\right)$ and $\left(\omega_{2}, \sigma_{2}\right)$ the corresponding configurations restricted to $\Lambda_{L}$. By the classical property of the total variation distance, there exists a coupling $\mathbf{Q}$ of $\mathbf{P}_{1}$ and $\mathbf{P}_{2}$ satisfying $\mathbf{Q}\left(\omega_{1}=\omega_{2}, \sigma_{1}=\sigma_{2}\right) \geq 1-\varepsilon$, and hence also $\mathbf{Q}\left(\omega_{1}=\omega_{2}, \sigma_{1}=\sigma_{2}, E_{l, L}\right) \geq 1-2 \varepsilon$. Note that conditioned on the latter event, by property (1) from Proposition 2.1, $\mathbf{S}$ and $\sigma_{1}$ have the same law when restricted to $\Lambda_{l}$. The last inequality therefore implies that the total variation distance between $\mathbf{S}$ and the marginal of $\mathbf{P}_{\mathbb{Z}^{2}}$ on $\sigma$ restricted to $\Lambda_{l}$ is smaller than $2 \varepsilon$. Since $l$ and $\varepsilon$ were arbitrary, this ends the proof.

We are now ready to prove our last theorem.

Proof of Theorem 2.8. For $i \in Q$, let $\operatorname{Perc}(i)$ be the event that the subgraph of $\mathbb{Z}^{2}$ induced by $\{v: \sigma(v)=i\}$ contains an infinite connected component. By Lemma 2.10 we know that $\sigma$ is distributed like an independent assignment of a spin to each cluster of $\omega$. By (2.3) all clusters of $\omega$ are finite almost surely, and hence $\operatorname{Perc}(i)$ are tail events with respect to these independent spin assignments. Therefore by Kolomogorov's 0-1 law, conditioned on $\omega$, the probability of $\operatorname{Perc}(i)$ is either 0 or 1 . In the latter case, by symmetry under spin relabelling, we have that the probability of $\bigcap_{i \in Q} \operatorname{Perc}(i)$ is also 1 . Hence,

$$
\begin{aligned}
\mathbf{P}_{\mathbb{Z}^{2}}\left(\bigcup_{i \in Q} \operatorname{Perc}(i)\right) & =\mathbf{E}_{\mathbb{Z}^{2}}\left[\mathbf{P}_{\mathbb{Z}^{2}}\left(\bigcup_{i \in Q} \operatorname{Perc}(i) \mid \omega\right)\right] \\
& =\mathbf{E}_{\mathbb{Z}^{2}}\left[\mathbf{P}_{\mathbb{Z}^{2}}\left(\bigcap_{i \in Q} \operatorname{Perc}(i) \mid \omega\right)\right] \\
& =\mathbf{P}_{\mathbb{Z}^{2}}\left(\bigcap_{i \in Q} \operatorname{Perc}(i)\right) .
\end{aligned}
$$

Note that for topological reasons, on the event $\operatorname{Perc}(i) \cap \operatorname{Perc}(j)$ for $i \neq j$, there must be at least one infinite interface in $\eta(\sigma)$ separating two infinite components with different $\sigma$-spins. Since $\eta(\sigma) \subseteq \omega^{\prime}$, we infer that on the event $\operatorname{Perc}(i) \cap \operatorname{Perc}(j)$, the configuration $\omega^{\prime}$ percolates. By self-duality and (2.3), $\omega^{\prime}$ does not percolate a.s., and therefore $\mathbf{P}_{\mathbb{Z}^{2}}(\operatorname{Perc}(i) \cap \operatorname{Perc}(j))=0$ for $i \neq j$. Hence by (2.6), $\mathbf{P}_{\mathbb{Z}^{2}}(\operatorname{Perc}(i))=0$ for all $i \in Q$. We therefore conclude that there are infinitely many clusters of $\eta(\sigma)$, and hence also of $\eta\left(\sigma^{\prime}\right)$, surrounding the origin a.s. Since $\omega$ does not percolate and $\eta\left(\sigma^{\prime}\right) \subseteq \omega$ a.s., there must be infinitely many clusters of $\omega$ surrounding the origin a.s. which gives (2.4).

It is now enough to use (2.4) to deduce delocalization of the height function (2.5). This can be done in a similar way to the proofs from Sect. 2.2. The arguments need to be adjusted to the topology of the torus by also considering noncontractible clusters that may intersect the path along which the increment of the height function is computed. We leave the details to the reader. 


\section{Relationship with known models}

For special values of the parameters $a, b$ and $q, q^{\prime}$, we recover various well known models of statistical mechanics. It will be useful to have an explicit formula for the probability of $\omega$ in terms of an associated Potts model. To this end recall the notation from the beginning of Section 1.

Corollary 3.1. The marginal distribution on $\left(\omega, \sigma^{\prime}\right)$ is given by

$$
\mathbf{P}\left(\omega, \sigma^{\prime}\right) \propto q^{k(\omega)} a^{\left|\eta\left(\sigma^{\prime}\right)\right|}(1-b)^{\left|\omega \backslash \eta\left(\sigma^{\prime}\right)\right|} b^{|\mathrm{E} \backslash \omega|} \mathbf{1}_{\left\{\eta\left(\sigma^{\prime}\right) \subseteq \omega\right\}}, \quad \sigma^{\prime} \in Q^{\prime \mathrm{U}}, \omega \subseteq \mathrm{E} .
$$

Summing over all $\sigma^{\prime}$, we get

$$
\mathbf{P}(\omega) \propto q^{k(\omega)}\left(\frac{1-b}{b}\right)^{|\omega|} Z_{(\mathrm{V}(\omega), \omega)^{*}, q^{\prime}}\left(\frac{1-a-b}{a}\right), \quad \omega \subseteq \mathrm{E} .
$$

Proof. The first equality follows directly from (2.1), and the fact that there are exactly $q^{k(\omega)}$ configurations of $\sigma$ which are constant on the clusters of $\omega$. We get the second equality from the fact that

$$
\sum_{\sigma^{\prime}: \eta\left(\sigma^{\prime}\right) \subseteq \omega}\left(\frac{a}{1-b}\right)^{\left|\eta\left(\sigma^{\prime}\right)\right|}=Z_{(\mathrm{V}(\omega), \omega)^{*}, q^{\prime}}\left(\frac{1-a-b}{a}\right) .
$$

Note that the Potts model whose partition function appears in (3.2) is ferromagnetic if and only if $a+b \leq 1$.

\subsection{FK-random cluster model for $a+b=1$}

Recall that in this case $\omega^{\prime}=\omega^{\dagger}$ almost surely, and hence (1.8) simplifies to

$$
\mathbf{P}\left(\omega, \omega^{\prime}, \sigma, \sigma^{\prime}\right)=\frac{1}{\mathcal{Z}} a^{|\omega|}(1-a)^{|\mathrm{E} \backslash \omega|}, \quad\left(\omega, \omega^{\prime}, \sigma, \sigma^{\prime}\right) \in \Omega \Sigma_{1},
$$

where $\Omega \Sigma_{1}=\Omega \Sigma_{\geq 1} \cap \Omega \Sigma_{\leq 1}$.

We first consider the case when $M$ is of genus zero. We can readily recognize the underlying Fortuin-Kasteleyn random cluster model [17] (see e.g. [21] for an exposition on this classical subject).

Proposition 3.2. Assume that $M$ is of genus zero, and $a+b=1$. Let

$$
p=\frac{q^{\prime}}{q^{\prime}+a^{-1}-1} .
$$

Let $k(\omega)$ be the number of clusters of $\omega$. Then the marginal distribution of $\mathbf{P}$ on $\omega$ is given by

$$
\mathbf{P}(\omega) \propto\left(q q^{\prime}\right)^{k(\omega)} p^{|\omega|}(1-p)^{|\mathrm{E} \backslash \omega|},
$$

which is the $\mathrm{FK}\left(q q^{\prime}\right)$ random cluster model measure on $\mathrm{G}$ with free boundary conditions.

Proof. Consider $\omega \subseteq \mathrm{E}$. Using (3.3), it is enough to count how many pairs of spin configurations $\left(\sigma, \sigma^{\prime}\right) \in \Sigma$ are compatible with $\omega$, meaning that $\left(\omega, \omega^{\dagger}, \sigma, \sigma^{\prime}\right) \in \Omega \Sigma_{1}$. By the definition of $\Omega \Sigma_{1}$, this is the same as requiring that $\sigma$ is constant on the connected components of $\omega$, and $\sigma^{\prime}$ is constant on the connected components of $\omega^{\dagger}$. Using Euler's formula

$$
k\left(\omega^{\dagger}\right)=k(\omega)+|\omega|-|V|+1
$$

we conclude that the total number of compatible pairs is $\left(q q^{\prime}\right)^{k(\omega)} q^{|\omega|} \times$ const, where const is independent of $\omega$. Plugging this into (3.3) we get

$$
\mathbf{P}(\omega) \propto\left(q q^{\prime}\right)^{k(\omega)}\left(a q^{\prime}\right)^{|\omega|}(1-x)^{|\mathrm{E} \backslash \omega|} \propto\left(q q^{\prime}\right)^{k(\omega)} p^{|\omega|}(1-p)^{|\mathrm{E} \backslash \omega|},
$$

which concludes the proof. 
Remark 3.3. For $q=q^{\prime}$, the point $a=b=1 / 2$ corresponds to $p=q /(q+1)$ which is the critical point of the $\mathrm{FK}\left(q^{2}\right)$ random cluster model on the square lattice [5].

Corollary 3.4. Assume that $M$ is of genus zero. Let $\omega$ be distributed according to the $\mathrm{FK}\left(q q^{\prime}\right)$ random cluster model with parameter $p$ as above. For each cluster of $\omega$, choose a spin $\sigma \in Q$, and for each cluster of $\omega^{\dagger}$, choose a spin $\sigma^{\prime} \in Q^{\prime}$ uniformly and independently of one another. Then $\left(\sigma, \sigma^{\prime}\right)$ has the distribution of the spin model (1.4) with $a$ as above and $a+b=1$.

Proof. This follows from the arguments in the proof above.

Remark 3.5. - For $q^{\prime}=1$ and $a$ arbitrary, there is no constraint on $\sigma$ and the coupling of $(\sigma, \omega)$ becomes the classical Edwards-Sokal coupling between the q-state Potts model and the $\mathrm{FK}(q)$ random cluster model [16].

- For $q=1, q^{\prime}=2$ and $a+b=1, \eta\left(\sigma^{\prime}\right)$ is an even subgraph of $\mathrm{G}$, meaning that the degree of every vertex in $\left(\mathrm{V}, \eta\left(\sigma^{\prime}\right)\right)$ is even, and $\omega$ is distributed like the $\mathrm{FK}(2)$ random cluster model. This coupling of $\left(\omega, \eta\left(\sigma^{\prime}\right)\right)$ is the same as in the work of Grimmett and Janson [22].

We now assume that $M$ is a torus. The necessary Euler's formula takes a slightly more complicated form in this case. We follow the notation of [10, Section 4.3.2]. Define $\delta(\omega) \in\{0,1,2\}$ depending on the topology of $\omega$ :

- if $\omega$ contains two non-contractible cycles of different homotopy, then $\delta(\omega)=2$;

- if $\omega$ contains a non-contractible cycle and all such cycles are homotopic, then $\delta(\omega)=$ 1 ;

- if all connected components of $\omega$ are contractible, then $\delta(\omega)=0$.

Note that $\delta(\omega)+\delta\left(\omega^{\dagger}\right)=2$. With this notation Euler's formula reads

$$
k\left(\omega^{\dagger}\right)=k(\omega)+|\omega|-\delta(\omega)-|\mathrm{V}|+1 .
$$

Using the same arguments as above, we can prove the following result.

Proposition 3.6. Assume that $M$ is a torus. Then the marginal distribution of $\mathbf{P}$ on $\omega$ is given by

$$
\mathbf{P}(\omega) \propto\left(q q^{\prime}\right)^{k(\omega)} q^{\prime-\delta(\omega)} p^{|\omega|}(1-p)^{|\mathbb{E} \backslash \omega|},
$$

where $p$ is an in Proposition 3.2.

In the case $q=q^{\prime}$ this distribution is that of the balanced random cluster model with parameter $q^{2}$ as defined in [10]. This model, unlike the standard random cluster model defined on a torus, exhibits duality meaning that if $\omega$ is distributed according to a balanced random cluster model measure, then so is $\omega^{\dagger}$. This is clear from the above result, as $\omega^{\prime}=\omega^{\dagger}$.

\subsection{The staggered six-vertex model for $q=q^{\prime}=2$}

Let $\mathrm{G}^{\times}$be the medial graph of $\mathrm{G}$ where a vertex is placed at the intersection of each primal edge and its dual, and where two vertices are adjacent if the corresponding pair of primal or dual edges share an endpoint. Note that the medial graph is 4-regular, and its faces are in a natural correspondence with $V \cup U$ - the vertices and faces of $G$. Moreover, the dual graph $\left(\mathrm{G}^{\times}\right)^{*}$ is bipartite since the faces of $\mathrm{G}^{\times}$corresponding to $\mathrm{V}$ can only be adjacent to the faces corresponding to $U$ and vice versa. Also note that the same medial graph is obtained if we start with $\mathrm{G}^{*}$ instead of $\mathrm{G}$. 


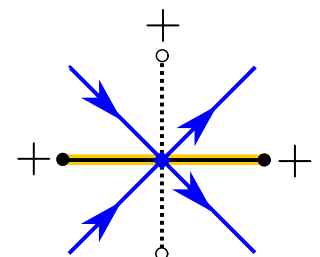

$1 \mathrm{a}-$

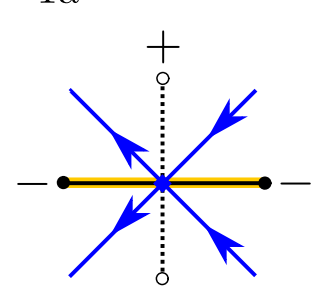

$1 \mathrm{~b}$

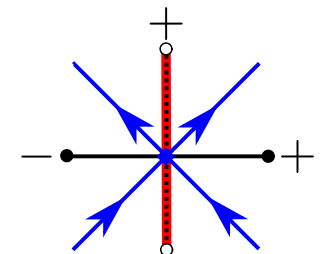

$2 \mathrm{a}+$

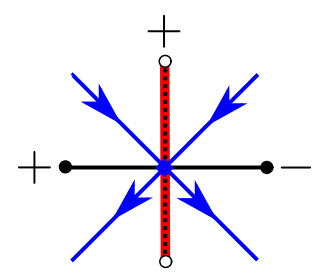

$2 \mathrm{~b}+$
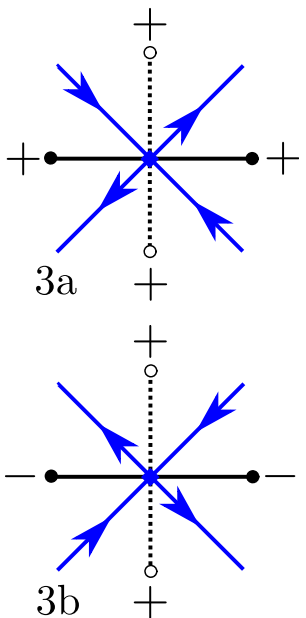

Figure 2: A primal edge (solid), its dual edge (dashed), and four corresponding medial edges (blue). The figure shows the three types (up to arrow reversal) of local arrow arrangements in the six-vertex model on the medial graph. The sets of yellow primal and red dual edges $\eta^{\prime}$ and $\eta$ are given by Rys' mapping. The signs are the values of $\tilde{\sigma}$ and $\tilde{\sigma}^{\prime}$ from (3.7). We have $\eta^{\prime}=\eta\left(\tilde{\sigma}^{\prime}\right)$ and $\eta=\eta(\tilde{\sigma})$

Let $\mathcal{O}$ be the set of assignments to each edge of $\mathrm{G}^{\times}$an orientation in such a way that there are exactly two incoming and two outgoing edges at each vertex of $\mathrm{G}^{\times}$. We say that an element of $\mathcal{O}$ is an arrow configuration. The (zero field) staggered six-vertex model with parameters $a, b>0$ (here we assume that the third parameter is $c=1$ ) is a probability measure on arrow configurations proportional to

$$
a^{N_{1}} b^{N_{2}},
$$

where $N_{1}$ and $N_{2}$ are the numbers of vertices of $\mathrm{G}^{\times}$with the local arrow arrangements of type 1 and 2 respectively $[25,26,33]$. The three types of local arrangements (each one has two subtypes) are pictured in Fig. 2.

An observable of interest in the six-vertex model is its height function $\tilde{H}$ defined on the faces of $G^{\times}$, or equivalently on $\mathrm{V} \cup \mathrm{U}$. It is given by first fixing its value at a chosen face $u_{0}$ of $\mathrm{G}^{\times}$. Then for any other face $u$, one draws a directed path $\gamma$ in the dual of $\mathrm{G}^{\times}$ (which is the quad graph of $\mathrm{G}$ and $\mathrm{G}^{*}$ ) connecting $u_{0}$ to $u$, and one defines $\tilde{H}_{\leftarrow}^{\gamma}(u)$ and $\tilde{H}_{\rightarrow}^{\gamma}(u)$ to be the numbers of arrows in the underlying six-vertex configuration that cross $\gamma$ from right to left, and from left to right respectively. The height at $u$ is then given by

$$
\tilde{H}(u)=\tilde{H}_{\leftarrow}^{\gamma}(u)-\tilde{H}_{\rightarrow}^{\gamma}(u) .
$$

In the case of genus zero, the right-hand side is independent of $\gamma$ since the surface is simply connected and six-vertex configurations form conservative flows. In higher genus the value of $H(u)$ in general depends on the homotopy class of the path $\gamma$, and can be thought of as a function defined on the faces of the universal cover of $G^{\times}$. Note that the height function has a fixed parity on $\mathrm{G}$ and $\mathrm{G}^{*}$.

We define $\mathcal{O}^{0}$ to be the set of arrow configurations for which the increment of the height function along any closed path in the dual of $G^{\times}$is equal to zero mod 4 . Note that $\mathcal{O}^{0}=\mathcal{O}$ if $M$ is of genus zero.

Rys in [38] introduced a correspondence between $\mathcal{O}^{0}$ and the set of pairs $\left(\eta, \eta^{\prime}\right)$ of certain primal and dual subgraphs that do not intersect (see Fig. 2). We note that 
this representation appeared also in the work of Nienhuis [31], and Boutillier and de Tilière [6] where it was used to represent the double Ising model as a free-fermion six-vertex model.

Lemma 3.7 (Rys' mapping). Let $q=q^{\prime}=2$. Then for each arrow configuration in $\mathcal{O}^{0}$, there exist exactly two spin configurations $\left(\tilde{\sigma}, \tilde{\sigma}^{\prime}\right) \in \Sigma$ that differ by a global sign change, such that $\eta=\eta(\tilde{\sigma})$, and $\eta^{\prime}=\eta\left(\tilde{\sigma}^{\prime}\right)$, where $\eta^{\prime}$ and $\eta$ are the sets of primal and dual edges respectively defined from the arrow configuration as in Fig. 2.

This correspondence is a consequence of the following construction. We chose to fix the height function $\tilde{H}$ to be \pm 1 with equal probability at a fixed face $u_{0} \in \mathrm{U}$. We now define spins $\tilde{\sigma}$ on $\mathrm{V}$ and $\tilde{\sigma}^{\prime}$ on $\mathrm{U}$ by

$$
\tilde{\sigma}(v)=i^{\tilde{H}(v)}, \quad \text { and } \quad \tilde{\sigma}^{\prime}(u)=i^{\tilde{H}(u)+1} .
$$

This definition depends only on the values of $\tilde{H} \bmod 4$, and since we consider only configurations from $\mathcal{O}^{0}, \tilde{\sigma}$ is a well defined function on the faces of $\mathrm{G}^{\times}$. We first claim that $\left(\tilde{\sigma}, \tilde{\sigma}^{\prime}\right) \in \Sigma$, which can be checked by inspection in Fig. 2 , where the spin $\tilde{\sigma}^{\prime}$ at the top was fixed to be +1 . On the other hand, knowing the spins $\left(\tilde{\sigma}, \tilde{\sigma}^{\prime}\right) \in \Sigma$ one can locally and consistently recover an arrow-configuration constructed as in Fig. 2.

Moreover, by our symmetric choice of $\tilde{H}\left(u_{0}\right)$ the distribution of $\left(\tilde{\sigma}, \tilde{\sigma}^{\prime}\right)$ is invariant under a global sign reversal, and since the weights from (3.5) agree with those from (1.4), we readily get the following correspondence.

Corollary 3.8. The law of $\left(\tilde{\sigma}, \tilde{\sigma}^{\prime}\right)$ induced by the map (3.7) from the law of the staggered six-vertex model on $\mathrm{G}^{\times}$conditioned on $\mathcal{O}^{0}$ has the same distribution as $\left(\sigma, \sigma^{\prime}\right)$ under $\mathbf{P}$. Moreover, the respective height functions $\tilde{H}$ and $H$ have the same distribution up to a global additive constant.

Remark 3.9. As mentioned before, our results from previous sections generalize to the case of nonhomogeneous weights $a_{e}$ and $b_{e}$ that depend on the particular edge. To obtain the classical (non-staggered) six-vertex model on the square lattice instead of the staggered one, one has to assign weights $a$ to the horizontal edges and $b$ to the vertical edges $\mathrm{G}$.

\subsection{Random currents for $q^{\prime}=2$ at the the free fermion point $a^{2}+b^{2}=1$}

Here we again assume that $M$ is of genus zero. A current is simply a function $\mathbf{n}: \mathrm{E} \rightarrow\{0,1, \ldots$,$\} . For a current \mathbf{n}$, define $\omega(\mathbf{n})$ and $\eta(\mathbf{n})$ to be the set of edges with non-zero and odd values of $\mathbf{n}$ respectively. In particular, $\eta \subseteq \omega$. We will say that the pair $(\eta(\mathbf{n}), \omega(\mathbf{n}))$ is the trace of the current $\mathbf{n}$. We will often identify a current with its trace as the trace contains all the relevant information for the probability measures that we will consider.

Let $\mathcal{E}$ be the collection of sets of edges $\eta$ such that each vertex in $\vee$ has even degree in the graph $(\mathrm{V}, \eta)$. Such $\eta$ are usually called even subgraphs of $\mathrm{G}$. By $\Gamma$ we will denote the set of all possible traces $(\omega, \eta)$ of currents such that $\eta \in \mathcal{E}$. The currents in $\Gamma$ are commonly called sourceless. Note that since $M$ is either a sphere or the plane, spin configurations $\sigma^{\prime}: U \rightarrow\{-1,+1\}$ are in a 2-to-1 correspondence with even subgraphs given by the map $\sigma^{\prime} \mapsto \eta\left(\sigma^{\prime}\right)$. This is not true for higher genera since then not all even subgraphs (e.g. a non-contractible cycle on a torus) can be realized as the set of interfaces of a spin configuration. In what follows we will write $\eta=\eta\left(\sigma^{\prime}\right)$.

\section{Single random current for $q=1$}

The single random current measure is induced from the power series expansion of the Ising model partition function (see e.g. [27]). We have the following observation: 
Corollary 3.10. Assume that $M$ is of genus zero. Let $a^{2}+b^{2}=1, q^{\prime}=2$ and $q=1$. Then

$$
\mathbf{P}(\eta, \omega) \propto a^{|\eta|}(1-b)^{|\omega \backslash \eta|} b^{|\Subset \backslash \omega|}, \quad(\eta, \omega) \in \Gamma,
$$

which is the law of the sourceless single random current with $a=\tanh J$.

Proof. The formula for the probability is a direct consequence of Corollary (3.1). The identification with the random current measure follows for instance from Lemma 3.1 of [27], by setting $a=\tanh J$ and $b=(\cosh J)^{-1}$.

\section{Double random current for $q=2$}

The double random current can be defined as the sum of two i.i.d. sourceless single random currents [20]. It turns out that going from a single current to a double current amounts to changing $q$ from 1 to 2 , and $J$ to $2 J$ :

Corollary 3.11. Assume that $M$ is of genus zero. Let $x \in(0,1]$ be given by $a=2 x /(1+$ $\left.x^{2}\right)$. Moreover, let $a^{2}+b^{2}=1$ and $q^{\prime}=q=2$. Then

$$
\mathbf{P}(\eta, \omega) \propto 2^{k(\omega)+|\omega|} x^{|\eta|}\left(x^{2}\right)^{|\omega \backslash \eta|}\left(1-x^{2}\right)^{|E \backslash \omega|}, \quad(\eta, \omega) \in \Gamma,
$$

which is the law of the sourceless double random current with $x=\tanh J$, or equivalently $a=\tanh 2 J$.

Proof. Again, the formula for the probability follows directly from Corollary (3.1). The identification with the double random current measure follows from Theorem 3.2 of [27], by setting $x=\tanh J$ and using the fact that since $a^{2}+b^{2}=1$, we have $b=$ $\left(1-x^{2}\right) /\left(1+x^{2}\right)$.

Remark 3.12. Recall that the XOR-Ising model is the pointwise product of two i.i.d. Ising model spin configurations $[6,9,40]$. It is known that in the free fermion case $a^{2}+b^{2}=1$, the distribution of $\sigma$ and $\sigma^{\prime}$ is that of the XOR-Ising model and its dual XORIsing model [6]. It follows from Corollary 3.11 and Proposition 2.1 that an independent assignment of $\mathrm{a} \pm 1$ spin to the clusters of a double random current yields the XORIsing model configuration. This result was, to our knowledge, first observed during a discussion of Roland Bauerschmidt, Hugo Duminil-Copin, Aran Raoufi and the author at IHES in 2017, and was the main inspiration for the considerations in this article.

\subsection{Loop $O(n)$ model for $q=n, q^{\prime}=2$ and $b=1$}

Assume that $M$ is of genus zero, and let $\mathrm{G}$ be a 3-regular graph e.g. a piece of the hexagonal lattice. For each spin configuration, $\sigma^{\prime}: U \rightarrow\{-1,1\}, \eta\left(\sigma^{\prime}\right)$ is a collection of disjoint loops on $\mathrm{G}$, and each such collection of loops corresponds to exactly two spin configurations. This implies that for $b=1$, the marginal distribution on $\eta=\eta\left(\sigma^{\prime}\right)$ is given by

$$
\mathbf{P}(\eta) \propto n^{k(\eta)} a^{|\eta|} \propto n^{\# \text { loops in } \eta} x^{|\eta|}, \quad \eta \in \mathcal{E},
$$

where $x=a / n$, and where $\mathcal{E}$ is the set of all collections of disjoint loops on $\mathrm{G}$. In the second identity, we used the fact that each vertex of $\mathrm{G}$ is either isolated in $(\mathrm{V}, \eta)$ or belongs to exactly two edges of $\eta$ This is the law of the loop $O(q)$ model with parameter $x$ (see e.g. [34] for an exposition on the subject).

Note that the spin model $\sigma^{\prime}$ for $q=1$ is the standard Ising model. We note that for real valued $n \geq 1$, this model has been studied in [15], where its FKG property (valid for $x \leq \frac{1}{\sqrt{n}}$ ) was used to prove existence of macroscopic loops at the Nienhuis' critical point. 


\section{5 $\sigma$ and $\sigma^{\prime}$ as classical spin models}

In this section we assume that $M$ is of genus zero. In this case the spin model $\sigma$ can be represented via a classical unconstrained spin system, which is a special case of the model of Domany and Riedel [8] where two Potts models are coupled via a general four-spin interaction. This property is a consequence of duality in the Potts model, and is a generalization to arbitrary $q$ and $q^{\prime}$ of the six-vertex model representation of the Ashkin-Teller model [31] (see also [6, 39]). We note that similar ideas applied to the model from [8] are present in [2].

We consider a spin model on configurations $\left(\mathrm{s}, \mathrm{s}^{\prime}\right) \in Q^{\mathrm{V}} \times{Q^{\prime}}^{\mathrm{V}}$ given by the GibbsBoltzmann distribution

$$
\tilde{\mu}\left(\mathbf{s}, \mathbf{s}^{\prime}\right) \propto \exp \left(\sum_{\left\{v_{1}, v_{2}\right\} \in \mathrm{E}} \delta_{\mathbf{s}\left(v_{1}\right), \mathbf{s}\left(v_{2}\right)}\left(\alpha+\beta \delta_{\mathbf{s}^{\prime}\left(v_{1}\right), \mathrm{s}^{\prime}\left(v_{2}\right)}\right)\right),
$$

where

$$
\alpha=\ln \left(\frac{1-a}{b}\right) \quad \text { and } \quad \beta=\ln \left(1+\frac{q^{\prime} a}{1-a}\right),
$$

and where $\delta_{x, y}=1$ if $x=y$ and $\delta_{x, y}=0$ otherwise. Note that $\beta$ is always positive, and $\alpha$ is positive if and only if $a+b \leq 1$. These parameters form a two-dimensional subspace of the parameters of the general model from [8].

Remark 3.13. This spin model can be defined on any finite graph, not necessarily embedded in a surface.

The following values of parameters are of special interest:

- The case $\alpha=0$, or equivalently $a+b=1$, corresponds to a ferromagnetic $q q^{\prime}$-state Potts model $\tilde{\mathrm{s}}$ with $J=\beta>0$. Then, $\mathrm{s}=\tilde{\mathrm{s}}(\bmod q)$ in distribution.

- For $q^{\prime}=2$, the case $\alpha=-\beta$, or equivalently $b-a=1$, on a bipartite graph corresponds to an antiferromagnetic $2 q$-state Potts model s̃ with $J=-\beta$. Indeed, if we flip the value of the $\mathrm{s}^{\prime}$ spin on all black vertices and call the resulting spin configuration $\mathrm{s}^{\prime \prime}$, we have $1-\delta_{\mathbf{s}^{\prime}\left(v_{1}\right), \mathrm{s}^{\prime}\left(v_{2}\right)}=\delta_{\mathrm{s}^{\prime \prime}\left(v_{1}\right), \mathrm{s}^{\prime \prime}\left(v_{2}\right)}$. Again, $\mathbf{s}=\tilde{\mathbf{s}}(\bmod q)$ in distribution.

- The case $2 \alpha=-\beta$, or $a^{2}+b^{2}=1$, results in symmetric energy levels $-\frac{\beta}{2}, 0, \frac{\beta}{2}$ per bond. For $q=q^{\prime}=2$, it corresponds to the free fermion point in the six-vertex model. In this case one can write the Hamiltonian as a sum of two independent Hamiltonians, and as a result represent the system as two statistically independent copies of the 2-state Potts model (the Ising model). For $q^{\prime}=2$ and $q>2$, the model is equivalent to the $q$-component version of the cubic model of Kim, Levy and Uffer [24].

Note that by definition (3.8), conditioned on the spins s, the spins $\mathrm{s}^{\prime}$ do not interact along edges with a different value of $s$ assigned to both endpoints. This is exactly the dual picture (zero coupling constant) of the hard-core constraint (1.3) (infinite coupling constant) for the spin model $\sigma$. Hence, the following identification of $\sigma$ and s which should not be surprising.

Proposition 3.14. Assume that $M$ is of genus zero. Then the distributions of $\sigma$ under $\mathbf{P}$, and of s under $\tilde{\mu}$ are the same.

The rest of the article is devoted to the proof of this result which is based on duality arguments. To this end, we need to recall the high-temperature expansion of the Potts model partition function and its planar duality. Let $G=(V, E)$ be a finite and not necessarily connected graph embedded in $M$, and let $G^{*}=\left(U, E^{*}\right)$ be its dual graph. 
We will often drop the parameter $q$ from the notation and write $Z_{G}$ for $Z_{G, q}$. Recall that $x+1=e^{J}$. We have

$$
\begin{aligned}
(x+1)^{|E|} Z_{G}(x) & =\sum_{\mathrm{s} \in Q^{V}} \exp \left(J \sum_{\left\{v_{1}, v_{2}\right\} \in E} \delta_{\mathrm{s}\left(v_{1}\right), \mathrm{s}\left(v_{2}\right)}\right) \\
& =\sum_{\mathbf{s} \in Q^{V}} \prod_{\left\{v_{1}, v_{2}\right\} \in E}\left(1+\delta_{\mathrm{s}\left(v_{1}\right), \mathrm{s}\left(v_{2}\right)} x\right) \\
& =\sum_{\mathbf{s} \in Q^{V}} \sum_{\xi \subseteq E} x^{|\xi|} \mathbf{1}\{\text { s constant on clusters of } \xi\} \\
& =\sum_{\xi \subseteq E} x^{|\xi|} q^{k(\xi)},
\end{aligned}
$$

where $k(\xi)$ is the number of clusters of $\xi$ in $G$, i.e., connected components of the graph $(V, \xi)$ including isolated vertices. Again recall that Euler's formula for planar graphs reads

$$
k(\xi)=f(\xi)-|\xi|+|V|-1,
$$

where $f(\xi)$ is the number of faces of $\xi$. Note that if we denote $\xi^{\dagger}=E^{*} \backslash \xi^{*}$, then $f(\xi)=k\left(\xi^{\dagger}\right)$ is the number of clusters of $\xi^{\dagger}$ in $G^{*}$. Hence by (3.10) we can write

$$
\begin{aligned}
Z_{G}(x) & =(x+1)^{-|E|} q^{|V|-1} \sum_{\xi \subseteq E}\left(\frac{x}{q}\right)^{|\xi|} q^{k\left(\xi^{\dagger}\right)} \\
& =q^{|V|-|E|-1}\left(\frac{x}{x+1}\right)^{|E|} \sum_{\xi^{\dagger} \subseteq E^{*}}\left(x^{*}\right)^{\mid \xi^{\dagger}} q^{k\left(\xi^{\dagger}\right)} \\
& =q^{|V|-|E|-1}\left(\frac{x}{x+1}\right)^{|E|}\left(x^{*}+1\right)^{\left|E^{*}\right|} Z_{G^{*}}\left(x^{*}\right) \\
& =q^{|V|-|E|-1}\left(\frac{x+q}{x+1}\right)^{|E|} Z_{G^{*}}\left(x^{*}\right),
\end{aligned}
$$

where $x^{*}=q / x$, and where in the third equality we again used (3.10).

Based on Corollary 3.1, we can now prove an intermediate result which gives a formula for the probability of $\omega$ in terms of the (primal) Potts model partition function $Z_{(\mathrm{V}, \omega), q^{\prime}}$.

Proposition 3.15. Assume that $M$ is of genus zero. Then, the marginal distribution of $\mathbf{P}$ on $\omega$ is given by

$$
\mathbf{P}(\omega) \propto q^{k(\omega)}\left(\frac{1-\left(1-q^{\prime}\right) a-b}{b}\right)^{|\omega|} Z_{(\mathrm{V}, \omega), q^{\prime}}\left(\frac{q^{\prime} a}{1-a-b}\right), \quad \omega \subseteq \mathrm{E} .
$$

Proof. Let $x^{*}=\frac{1-a-b}{a}$ and $x=\frac{q^{\prime}}{x^{*}}=\frac{q^{\prime} a}{1-a-b}$, and note that

$$
Z_{(\mathrm{V}(\omega), \omega)}=q^{|V(\omega)|-|\mathrm{V}|} Z_{(\mathrm{V}, \omega)} .
$$

Applying duality we get

$$
Z_{(\mathrm{V}(\omega), \omega)}(x)=q^{\prime|V(\omega)|-|\omega|-1}\left(\frac{x+q^{\prime}}{x+1}\right)^{|\omega|} Z_{(\mathrm{V}(\omega), \omega)^{*}}\left(x^{*}\right) .
$$

Therefore, by Corollary 3.1 we have

$$
\begin{aligned}
q^{-k(\omega)}\left(\frac{b}{1-b}\right)^{|\omega|} \mathbf{P}(\omega) & \propto Z_{(\mathrm{V}(\omega), \omega)^{*}}\left(x^{*}\right) \\
& =q^{-|V(\omega)|+|\omega|+1}\left(\frac{x+1}{x+q^{\prime}}\right)^{|\omega|} Z_{(\mathrm{V}(\omega), \omega)}(x) \\
& =q^{-|V(\omega)|+|\omega|+1+|V(\omega)|-|\mathrm{V}|}\left(\frac{x+1}{x+q^{\prime}}\right)^{|\omega|} Z_{(\mathrm{V}, \omega)}(x) \\
& =\left(\frac{1-\left(1-q^{\prime}\right) a-b}{1-b}\right)^{|\omega|} Z_{(\mathrm{V}, \omega)}(x),
\end{aligned}
$$

where in the last equality we used the identity $q^{\prime} \frac{x+1}{x+q^{\prime}}=\frac{1-\left(1-q^{\prime}\right) a-b}{1-b}$. 
Note that for $a+b>1$ and $b \leq 1$, we have that $x<-1$, and hence the Potts model corresponding to the partition function $Z_{(\mathrm{V}, \omega), q^{\prime}}(x)$ has a complex-valued coupling constant $J$. To complete the proof of Proposition 3.14 we need the following elementary lemma which effectively turns this coupling constant into a real-valued one.

Lemma 3.16. Let $G=(V, E)$ be a finite graph. Then for all $t \neq-1$, we have

$$
\sum_{\xi \subseteq E} t^{|\xi|}(x+1)^{|\xi|} Z_{(V, \xi)}(x)=(1+t(x+1))^{|E|} Z_{G}\left(\frac{t x}{1+t}\right) .
$$

Proof. We have

$$
\begin{aligned}
\sum_{\xi \subseteq E} t^{|\xi|}(x+1)^{|\xi|} Z_{(V, \xi)}(x) & =\sum_{\xi \subseteq E} t^{|\xi|} \sum_{\xi^{\prime} \subseteq \xi} x^{\left|\xi^{\prime}\right|} q^{k\left(\xi^{\prime}\right)} \\
& =\sum_{\xi^{\prime} \subseteq E} \sum_{\xi \supseteq \xi^{\prime}} t^{|\xi|} x^{\left|\xi^{\prime}\right|} q^{k\left(\xi^{\prime}\right)} \\
& =\sum_{\xi^{\prime} \subseteq E}(1+t)^{|E|-\left|\xi^{\prime}\right|}(t x)^{\left|\xi^{\prime}\right|} q^{k\left(\xi^{\prime}\right)} \\
& =(1+t)^{|E|} \sum_{\xi^{\prime} \subseteq E}\left(\frac{t x}{1+t}\right)^{\left|\xi^{\prime}\right|} q^{k\left(\xi^{\prime}\right)} \\
& =(1+t(x+1))^{|E|} Z_{G}\left(\frac{t x}{1+t}\right),
\end{aligned}
$$

where in the first and last equality we applied the high-temperature expansion (3.10).

We can finally identify the distribution of $\sigma$ as that of s.

Proof of Proposition 3.14. For $i \in Q$ and $\mathrm{s} \in Q^{\mathrm{V}}$, we define

$$
E_{i}(\mathbf{s})=\left\{\left\{v_{1}, v_{2}\right\} \in \mathrm{E}: \mathbf{s}\left(v_{1}\right)=\mathbf{s}\left(v_{2}\right)=i\right\} \quad \text { and } \quad E(\mathbf{s})=\bigcup_{i \in Q} E_{i}(\mathbf{s})
$$

For $\xi \subseteq \mathrm{V}$, we denote $\tilde{Z}_{\xi}=Z_{(\mathrm{V}(\xi), \xi), q^{\prime}}$. On one hand, by the definition of the spin model (3.8), conditioned on $s$, the $\mathrm{s}^{\prime}$ spins do not interact whenever the corresponding $s$ spins are different, and hence we have

$$
\begin{aligned}
\tilde{\mu}(\mathrm{s}) & \propto\left(q^{\prime}\right)^{|\mathrm{V}|-|V(E(\mathrm{~s}))|} e^{\alpha|E(\mathrm{~s})|} \prod_{i \in Q} e^{\beta\left|E_{i}(\mathrm{~s})\right|} \tilde{Z}_{E_{i}(\mathrm{~s})}\left(e^{\beta}-1\right) \\
& =\left(q^{\prime}\right)^{|\mathrm{V}|-|V(E(\mathrm{~s}))|} e^{(\alpha+\beta)|E(\mathrm{~s})|} \prod_{i \in Q} \tilde{Z}_{E_{i}(\mathrm{~s})}\left(\frac{q^{\prime} a}{1-a}\right) \\
& =\left(q^{\prime}\right)^{|\mathrm{V}|-|V(E(\mathrm{~s}))|}\left(\frac{1-\left(1-q^{\prime}\right) a}{b}\right)^{|E(\mathrm{~s})|} \prod_{i \in Q} \tilde{Z}_{E_{i}(\mathrm{~s})}\left(\frac{q^{\prime} a}{1-a}\right) .
\end{aligned}
$$

On the other hand, by property (1) from Proposition 2.1, and Proposition 3.15, we have

$$
\begin{aligned}
\mathbf{P}(\sigma) & \propto \sum_{\omega \text { consistent with } \sigma}\left(\frac{1-\left(1-q^{\prime}\right) a-b}{b}\right)^{|\omega|} Z_{(\mathrm{V}, \omega)}\left(\frac{q^{\prime} a}{1-a-b}\right) \\
& =\sum_{\omega \text { consistent with } \sigma}\left(\frac{1-\left(1-q^{\prime}\right) a-b}{b}\right)^{|\omega|}\left(q^{\prime}\right)^{|\mathrm{V}|-|V(\omega)|} \prod_{i \in Q} \tilde{Z}_{E_{i}(\sigma) \cap \omega}\left(\frac{q^{\prime} a}{1-a-b}\right) \\
& =\left(q^{\prime}\right)^{|\mathrm{V}|-|V(E(\sigma))|} \prod_{i \in Q} \sum_{\xi \subseteq E_{i}(\sigma)}\left(\frac{1-\left(1-q^{\prime}\right) a-b}{b}\right)^{|\xi|}\left(q^{\prime}\right)^{\left|V\left(E_{i}(\sigma)\right)\right|-|V(\xi)|} \tilde{Z}_{\xi}\left(\frac{q^{\prime} a}{1-a-b}\right) \\
& =\left(q^{\prime}\right)^{|\mathrm{V}|-|V(E(\sigma))|}\left(\frac{1-\left(1-q^{\prime}\right) a}{b}\right)^{|E(\mathrm{~s})|} \prod_{i \in Q} \tilde{Z}_{E_{i}(\mathrm{~s})}\left(\frac{q^{\prime} a}{1-a}\right)
\end{aligned}
$$

where ' $\omega$ consistent with $\sigma$ ' means that $\sigma$ is constant on the clusters of $\omega$, and where in the last equality we used Lemma 3.16 with $t=(1-a-b) / b$ and $x=q^{\prime} a /(1-a-b)$. The formulas (3.15) and (3.16) are identical and we finish the proof. 
Spins, percolation and height functions

\section{References}

[1] J. Ashkin and E. Teller, Statistics of two-dimensional lattices with four components, Phys. Rev. 64 (1943Sep), 178-184.

[2] H. Au-Yang and J. H. H. Perk, Solutions of the star-triangle equations in $\left(n_{\alpha}, n_{\beta}\right)$ potts models, International Journal of Modern Physics A 7 (1992), no. supp01b, 1025-1046. MR1187587

[3] R. J. Baxter, S. B. Kelland, and F. Y. Wu, Equivalence of the Potts model or Whitney polynomial with an ice-type model, Journal of Physics A: Mathematical and General 9 (1976), no. 3, 397.

[4] R. J. Baxter, Exactly solved models in statistical mechanics, Academic Press Inc. [Harcourt Brace Jovanovich Publishers], London, 1982. MR690578 (86i:82002a)

[5] V. Beffara and H. Duminil-Copin, The self-dual point of the two-dimensional random-cluster model is critical for $q \geq 1$, Probability Theory and Related Fields 153 (2012Aug), no. 3, 511-542. MR2948685

[6] C. Boutillier and B. de Tilière, Height representation of XOR-Ising loops via bipartite dimers, Electron. J. Probab. 19 (2014), no. 80, 33. MR3256880

[7] N. Chandgotia, R. Peled, S. Sheffield, and M. Tassy, Delocalization of uniform graph homomorphisms from $\mathbb{Z}^{2}$ to $\mathbb{Z}$, Communications in Mathematical Physics 387 (2021), no. 2, 621-647. MR4315657

[8] E. Domany and E. K. Riedel, Phase transitions in two-dimensional systems, Journal of Applied Physics 49 (1978), no. 3, 1315-1320.

[9] J. Dubédat, Exact bosonization of the Ising model, 2011. arXiv:1112.4399.

[10] H. Duminil-Copin, Parafermionic Observables and Their Applications to Planar Statistical Physics Models, Ensaios Matemáticos 25, Sociedade Brasileira de Matemática, 2014. MR3184487

[11] H. Duminil-Copin, M. Gagnebin, M. Harel, I. Manolescu, and V. Tassion, Discontinuity of the phase transition for the planar random-cluster and Potts models with $q>4$. arXiv:1611.09877. MR4371394

[12] H. Duminil-Copin and M. Lis, On the double random current nesting field, Probability Theory and Related Fields (2019Mar). MR4026609

[13] H. Duminil-Copin, M. Lis, and W. Qian, Conformal invariance of double random currents I: identification of the limit, 2021. arXiv:2107.12985.

[14] H. Duminil-Copin, M. Lis, and W. Qian, Conformal invariance of double random currents II: precompactness and properties in the discrete, 2021. arXiv:2107.12880.

[15] H. Duminil-Copin, A. Glazman, R. Peled, and Y. Spinka, Macroscopic loops in the loop o(n) model at nienhuis' critical point, Journal of the European Mathematical Society 23 (2020), no. 1, 315-347. MR4186469

[16] R. G. Edwards and A. D. Sokal, Generalization of the Fortuin-Kasteleyn-Swendsen-Wang representation and Monte Carlo algorithm, Phys. Rev. D 38 (1988Sep), 2009-2012. MR0965465

[17] C. M. Fortuin and P. W. Kasteleyn, On the random-cluster model: I. Introduction and relation to other models, Physica 57 (1972), no. 4, 536-564. MR0359655

[18] A. Giuliani, V. Mastropietro, and F. L. Toninelli, Height fluctuations in interacting dimers, Annales de l’Institut Henri Poincaré, Probabilités et Statistiques 53 (2017), no. 1, 98-168. MR3606736

[19] A. Glazman and R. Peled, On the transition between the disordered and antiferroelectric phases of the 6-vertex model, 2018. arXiv:1909.03436.

[20] R. B. Griffiths, C. A. Hurst, and S. Sherman, Concavity of Magnetization of an Ising Ferromagnet in a Positive External Field, Journal of Mathematical Physics 11 (1970), no. 3, 790-795. MR0266507

[21] G. Grimmett, The random-cluster model, Probability on discrete structures, 2004, pp. 73-123. MR2023651

[22] G. Grimmett and S. Janson, Random even graphs., Electron. J. Comb. 16 (2009), no. 1 (English). MR2491648 
Spins, percolation and height functions

[23] R. Kenyon, Dominos and the Gaussian free field, Ann. Probab. 29 (2001), no. 3, 1128-1137. MR1872739

[24] D. Kim, P. M. Levy, and L. F. Uffer, Cubic rare-earth compounds: Variants of the three-state Potts model, Physical Review B 12 (1975), no. 3, 989.

[25] E. H. Lieb, Exact solution of the $f$ model of an antiferroelectric, Phys. Rev. Lett. 18 (1967Jun), 1046-1048.

[26] E. H. Lieb, Residual entropy of square ice, Phys. Rev. 162 (1967Oct), 162-172.

[27] M. Lis, The planar Ising model and total positivity, J. Stat. Phys. 166 (2017), no. 1, 72-89. MR3592851

[28] M. Lis, On Boundary Correlations in Planar Ashkin-Teller Models, International Mathematics Research Notices (2021). rnaa380.

[29] M. Lis, On delocalization in the six-vertex model, Communications in Mathematical Physics 383 (2021), no. 2, 1181-1205. MR4239840

[30] T. Lupu and W. Werner, A note on Ising random currents, Ising-FK, loop-soups and the Gaussian free field, Electron. Commun. Probab. 21 (2016), 7 pp. MR3485382

[31] B. Nienhuis, Critical behavior of two-dimensional spin models and charge asymmetry in the Coulomb gas, Journal of Statistical Physics 34 (1984Mar), no. 5, 731-761. MR0751711

[32] A. L. Owczarek and R. J. Baxter, A class of interaction-round-a-face models and its equivalence with an ice-type model, Journal of Statistical Physics 49 (1987Dec), no. 5, 1093-1115. MR0935487

[33] L. Pauling, The structure and entropy of ice and of other crystals with some randomness of atomic arrangement, Journal of the American Chemical Society 57 (1935), no. 12, 2680-2684.

[34] R. Peled and Y. Spinka, Lectures on the Spin and Loop O(n) Models, 2017. To appear in "Sojourns in Probability Theory and Statistical Physics", celebrating Chuck Newman's 70th birthday.

[35] C. E. Pfister and Y. Velenik, Random-cluster representation of the Ashkin-Teller model, Journal of Statistical Physics 88 (1997Sep), no. 5, 1295-1331. MR1478070

[36] R. B. Potts, Some generalized order-disorder transformations, Mathematical Proceedings of the Cambridge Philosophical Society 48 (1952), no. 1, 106-109. MR0047571

[37] G. Ray and Y. Spinka, Finitary codings for gradient models and a new graphical representation for the six-vertex model, 2019. arXiv:1908.09056.

[38] F. Rys, Über ein zweidimensionales klassisches Konfigurationsmodell, Helvetica Physica Acta 36 (1963).

[39] F. Wegner, Duality relation between the Ashkin-Teller and the eight-vertex model, Journal of Physics C: Solid State Physics 5 (1972), no. 11, L131.

[40] D. B. Wilson, XOR-Ising Loops and the Gaussian Free Field, 2011. arXiv:1102.3782.

Acknowledgments. I am grateful to Roland Bauerschmidt, Hugo Duminil-Copin and Aran Raoufi for the discussions on the double random current and XOR-Ising model that we had in 2017 at IHES, Bures-sur-Yvette, and that were the inspiration for this work. I also thank Alexander Glazman and Ron Peled for their very useful comments and suggestions, and Jacques H.H. Perk for bringing to my attention the model of Domany and Riedel [8] 


\section{Electronic Journal of Probability Electronic Communications in Probability}

\section{Advantages of publishing in EJP-ECP}

- Very high standards

- Free for authors, free for readers

- Quick publication (no backlog)

- Secure publication $\left(\mathrm{LOCKSS}^{1}\right)$

- Easy interface (EJMS²)

\section{Economical model of EJP-ECP}

- Non profit, sponsored by $\mathrm{IMS}^{3}, \mathrm{BS}^{4}$, ProjectEuclid ${ }^{5}$

- Purely electronic

\section{Help keep the journal free and vigorous}

- Donate to the IMS open access fund ${ }^{6}$ (click here to donate!)

- Submit your best articles to EJP-ECP

- Choose EJP-ECP over for-profit journals

\footnotetext{
${ }^{1}$ LOCKSS: Lots of Copies Keep Stuff Safe http://www. lockss.org/

${ }^{2}$ EJMS: Electronic Journal Management System http://www.vtex.lt/en/ejms.html

${ }^{3}$ IMS: Institute of Mathematical Statistics http://www.imstat.org/

${ }^{4}$ BS: Bernoulli Society http://www. bernoulli-society.org/

${ }^{5}$ Project Euclid: https://projecteuclid.org/

${ }^{6}$ IMS Open Access Fund: http://www.imstat.org/publications/open.htm
} 